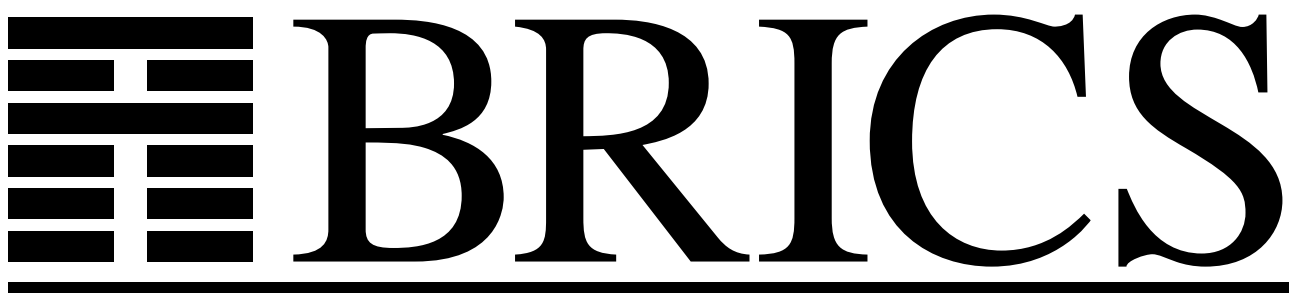

Basic Research in Computer Science

\title{
On the Recursive Enumerability of Fixed-Point Combinators
}

Mayer Goldberg

RS-05-1 
Copyright c 2005, Mayer Goldberg.

BRICS, Department of Computer Science University of Aarhus. All rights reserved.

Reproduction of all or part of this work is permitted for educational or research use on condition that this copyright notice is included in any copy.

See back inner page for a list of recent BRICS Report Series publications. Copies may be obtained by contacting:

\author{
BRICS \\ Department of Computer Science \\ University of Aarhus \\ Ny Munkegade, building 540 \\ DK-8000 Aarhus C \\ Denmark \\ Telephone: +4589423360 \\ Telefax: $\quad+4589423255$ \\ Internet: BRICS@brics.dk
}

BRICS publications are in general accessible through the World Wide Web and anonymous FTP through these URLs:

http://www.brics.dk

ftp: //ftp.brics.dk

This document in subdirectory RS / $05 / 1$ / 


\title{
On the Recursive Enumerability of Fixed-Point Combinators
}

\author{
Mayer Goldberg*
}

January, 2005

\begin{abstract}
We show that the set of fixed-point combinators forms a recursivelyenumerable subset of a larger set of terms we call non-standard fixedpoint combinators. These terms are observationally equivalent to fixedpoint combinators in any computable context, but the set of non-standard fixed-point combinators is not recursively enumerable.
\end{abstract}

\section{Introduction}

Fixed-point combinators are considered classical material by now in the $\lambda$ calculus, combinatory logic, and functional programming. They are used to define recursive functions and circular data structures by replacing recursion and circularity with self-application. Many fixed-point combinators are known, including ones by Curry [3, Page 178], Turing [7], and Klop [1]. A well-known construction due to Böhm is used to show that there are infinitely-many distinct fixed-point combinators [6].

In this paper we show that the set of fixed-point combinators is recursively enumerable. We found the implications of this result quite surprising: If we think of a fixed-point combinator as a device for defining recursive functions, then the existence of a partial recursive function that identifies fixed-point combinators suggests that we may be able to identify, either statically or dynamically, certain classes of $\lambda$-terms as having a potential for non-termination, given that they reduce to terms that contain a fixed-point combinator as a sub-expression.

The second result presented in this paper sets the theoretical limit for this endeavor: It has been known for some time that there exist terms that have the Böhm-tree of a fixed-point combinator, but that in fact fail to satisfy the equation that defines a fixed-point combinator [5]. Such terms can however be used to define recursive functions and circular structures in just the same

* Department of Computer Science, Ben Gurion University, P.O. Box 653, Beer Sheva 84105, Israel. 
way as ordinary fixed-point combinators are used. These curious terms are indistinguishable from fixed-point combinators in any applicative context. Put otherwise, such terms are observationally equivalent to fixed-point combinators. We show that the set of fixed-point combinators forms a recursively enumerable subset of a larger set that is not recursively enumerable. We refer to terms in this larger set as non-standard fixed-point combinators, and terms that fail to satisfy the fixed-point equation as strictly non-standard fixed-point combinators.

\subsection{Notation and Pre-requisites}

This work is carried out in the $\lambda \mathbf{K} \beta \eta$-calculus [1]. The $=$ symbol on $\lambda$-terms is taken to mean textual identity of language tokens, unless otherwise stated (e.g., taken modulo $\alpha$-conversion). The one-step $\beta \eta$-reduction is given by $\longrightarrow \beta \eta$, and the equivalence relation induced by the $\beta \eta$-relation is given by $=_{\beta \eta}$. Equality "by definition" is given by $\equiv$. The set of $\lambda$-terms is given by $\Lambda$. The set of combinators, given by $\Lambda^{0}$, is the set of terms with no free variables (aka "closed terms"). We use Church numerals $[1,2]$ to do arithmetic in the $\lambda$-calculus. The $n$-th Church numeral is given by $c_{n}$. The successor function on Church numerals is given by Succ. The logical truth values False, True are modeled in the $\lambda$-calculus using the combinators:

$$
\begin{aligned}
\text { False } & =\lambda x y \cdot y \\
\text { True } & =\lambda x y \cdot x
\end{aligned}
$$

For any $\lambda$-term $F$, the fixed point of $F$ is a $\lambda$-term $x$ such that $F x=\beta \eta x$. A fixed-point combinator $[1$, Section 6.1$]$ is a $\lambda$-term with the property that when applied to any $\lambda$-term $x$ it returns the fixed point of $x$. More formally, a $\lambda$-term $\Phi$ is a fixed-point combinator if for any $\lambda$-term $x$, the following holds:

$$
\Phi x \quad{ }_{\beta \eta} \quad x(\Phi x)
$$

The set $\mathbb{N}$ is the set of natural numbers not including zero. We use the notation $\{0\} \cup \mathbb{N}$ to refer to the set of non-negative integers.

\section{The set of fixed-point combinators is recur- sively enumerable}

We present a construction enumerating the set of fixed-point combinators. A similar construction can be shown for sets of $n$ multiple fixed-point combinators, for any $n>1$.

\subsection{TheOREM: The set of fixed-point combinators is recursively enumerable.}

Proof: A combinator $\Phi$ is a fixed-point combinator if for any term $x \in \Lambda$ we have $\Phi x={ }_{\beta \eta} x(\Phi x)$. By the $\xi$-rule [1, Page 23], we abstract over $x$ to get $\lambda x . \Phi x={ }_{\beta \eta} \lambda x . x(\Phi x)$. By $\eta$-reducing the left-hand side we get $\Phi={ }_{\beta \eta} \lambda x . x(\Phi x)$. 
Therefore, by the Church-Rosser theorem, there exist $n, m \in \mathbb{N}, \Psi \in \Lambda$, such that

$$
\Phi x . x(\Phi x) \underbrace{\longrightarrow \beta \eta \cdots \longrightarrow \beta \eta}_{n} \Psi
$$

This equality can be verified mechanically, terminating if the equality holds, and diverging otherwise. This, together with the fact that the set $\Lambda^{0}$ (of combinators) is recursively enumerable, implies that the set of fixed-point combinators is recursively enumerable. A detailed construction follows.

The set $\Lambda^{0}$ is recursively enumerable, so there exists an effective surjection $\mathfrak{C}: \mathbb{N} \rightarrow \Lambda^{0}$. Let $V_{0}=\emptyset$. For any $j>0$ we define:

$$
\begin{aligned}
C_{j} & \equiv \mathfrak{C}(j) \\
D_{j} & \equiv \lambda x \cdot x\left(C_{j} x\right)
\end{aligned}
$$

If we can show that $C_{j}={ }_{\beta \eta} D_{j}$, then it follows that $C_{j}$ is a fixed-point combinator. To do this "in parallel", for $j=1,2, \ldots$, we introduce the structure $Q_{j}$ :

$$
Q_{j}=\left\langle C_{j},\left\{C_{j}\right\},\left\{D_{j}\right\}, j\right\rangle
$$

We use this structure to maintain all the terms that can be reached from $C_{j}$ and $D_{j}$ via any finite number of $\beta \eta$-steps, so as we move from $V_{j}$ to $V_{j+1}$, the second and third projections of each of the quadruples "grow" to include all terms that can be reached by an additional $\beta \eta$-step. We now define the set $F_{j}$ of all quadruples for which we can now show that the first projection is a fixed-point combinator:

$$
F_{j}=\left\{\left\langle x_{1}, x_{2}, x_{3}, x_{4}\right\rangle \in V_{j}: x_{2} \cap x_{3} \neq \emptyset\right\}
$$

Note that for all quadruples in $F_{j}$ (and indeed in $V_{j}$ as well), the relation between the first and fourth projections is given by $x_{1}=\mathfrak{C}\left(x_{4}\right)$. Consequently, there is a unique ordering on the quadruples in $F_{j}$ that is induced by the ascending order of the fourth projection of each quadruple. The first projection of any quadruple in $F_{j}$ is a fixed-point combinator, and we list these fixed-point combinators in the ordering induced by the ordering on the quadruples. We now remove the structures that correspond to fixed-point combinators:

$$
W_{j}=V_{j} \backslash F_{j}
$$

And extend the second and third projections respectively, to include all the terms that can be reached after one additional $\beta \eta$-step:

$$
\begin{aligned}
& W_{j}^{\prime}=\left\{\left\langle x_{1}, x_{2}^{\prime}, x_{3}^{\prime}, x_{4}\right\rangle:\right. \\
&\left\langle x_{1}, x_{2}, x_{3}, x_{4}\right\rangle \in W_{j}, \\
& x_{2}^{\prime}=x_{2} \cup\left\{y: x \rightarrow_{\beta \eta} y, \text { for some } x \in x_{2}\right\} \\
&\left.x_{3}^{\prime}=x_{3} \cup\left\{y: x \rightarrow_{\beta \eta} y, \text { for some } x \in x_{3}\right\}\right\}
\end{aligned}
$$


The set $V_{j+1}$ is defined to contain both the new structure $Q_{j}$, as well as the extended quadruples:

$$
V_{j+1}=\left\{Q_{j}\right\} \cup W_{j}^{\prime}
$$

The enumeration of the set of fixed-point combinators is obtained by enumerating the first projections in all quadruples in $\left\{F_{j}\right\}_{j \in \mathbb{N}}$ according to the ordering induced by $j$, as the primary index, and then by the fourth projections in each $F_{j}$, as the secondary index.

A similar argument is used to show the recursively enumerable of the set of fixed points for any given combinator:

2.2 Corollary: For any combinator $F$, the set of fixed points of $F$, i.e., the set $\left\{x: F x={ }_{\beta \eta} x\right\}$ is recursively enumerable.

Proof: The construction is essentially the same as in the proof of Theorem 2.1, but with a different definition of $D_{j}: D_{j} \equiv F C_{j}$.

\section{Non-standard fixed-point combinators}

All fixed-point combinators have the same infinite extension $\lambda f . f(f(f \cdots))$, which is the same as stating they all have the same Böhm tree [1, Page 217]:

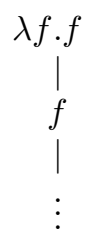

The converse does not hold, i.e., it is not the case that any term with the infinite extension $\lambda f . f(f(f \cdots))$ is a fixed-point combinator. Statman gives an example of such a term in his paper Some Examples of Non-Existent Combinators [5, Page 442], where for $M \equiv \lambda x . x x, B \equiv \lambda x y z . x(y z)$, he makes the observation that:

Note that $B M(B(B M) B)$ has the right Böhm tree to be a fixed point combinator but it is not one.

The term pointed out by Statman is one of many similar terms that fail to satisfy Equation (1) for any finite $m, n$. Such terms, however, can be used to define recursive functions. This motivates the following definition.

3.1 Definition: A non-standard fixed-point combinator. A term $\Psi$ is a non-standard fixed-point combinator if $\Psi$ has the same Böhm-tree as a fixed-point combinator.

Clearly, the set of fixed-point combinators is a proper subset of the set of non-standard fixed-point combinators. We therefore refer to non-standard fixedpoint combinator that is not a fixed-point combinator as a strictly non-standard fixed-point combinator. 
The above definition can be extended to sets of $n$ multiple fixed-point combinators, for $n>1$.

We can define a non-standard fixed-point combinator $\Psi$ by abstracting $f$ over an expression $E_{1}$ that reduces to an application of $f$ over an expression $E_{2}$, etc., so that $\Psi$ has the infinite extension $\lambda f . f(f(f \cdots))$. For $\Psi$ to be a strictly non-standard fixed-point combinator, the set of terms $\left\{E_{n}\right\}_{n \in \mathbb{N}}$ must satisfy $\neg\left(E_{n}=_{\beta \eta} E_{n+1}\right)$ for all $n \in \mathbb{N}$.

We define our own construction for a non-standard fixed-point combinator as follows:

$$
\begin{gathered}
\Psi \quad \lambda f .((\lambda x n \cdot f(x x(\text { Succ } n))) \\
(\lambda x n \cdot f(x x(\text { Succ } n))) \\
\left.c_{1}\right)
\end{gathered}
$$

The corresponding $E_{n}, E_{n+1}$ are as follows:

$$
\begin{aligned}
E_{n}= & ((\lambda x n . f(x x(\text { Succ } n))) \\
& (\lambda x n . f(x x(\text { Succ } n))) \\
& \left.c_{n}\right) \\
E_{n+1}= & ((\lambda x n . f(x x(\text { Succ } n))) \\
& (\lambda x n . f(x x(\text { Succ } n))) \\
& \left.c_{n+1}\right)
\end{aligned}
$$

and cannot be shown to be equal within a finite number of $\beta \eta$-steps.

The definition of $\Psi$ may appear contrived, since it makes no real use of $n$, and any good compiler, e.g., for Scheme or Common LISP, would optimize it away, reducing $\Psi$ to Curry's well-known fixed-point combinator. It is not difficult, however, to force $n$ to do some useful work, and thus prevent its elimination by an optimizing compiler. For example, we could apply it to $f$, as in this alternate non-standard fixed-point combinator:

$$
\begin{gathered}
\Psi^{\prime} \equiv \lambda f .((\lambda x n . n f(x x(\operatorname{Succ} n))) \\
(\lambda x n . n f(x x(\text { Succ } n))) \\
\left.c_{1}\right)
\end{gathered}
$$

3.2 THEOREM: The set of non-standard fixed-point combinators is not recursively enumerable.

Proof: Let $\mathrm{S}$ be the set of non-standard fixed-point combinators. The structure of our proof is as follows:

- We show that $\mathrm{S}$ is not recursive.

- We show that the complement of $\mathrm{S}$, given by $\overline{\mathrm{S}}=\Lambda^{0} \backslash \mathrm{S}$, is recursively enumerable.

It follows from the above two items that $\mathrm{S}$ is not recursively enumerable, for if both $S, \bar{S}$ are recursively enumerable, then both are recursive [4, Page 58]. The details of the proof follow. 
If $\mathrm{S}$ is recursive, then there exists a combinator $E$, such that for any combinator $M$, with encoding $\ulcorner M\urcorner$, we have:

$$
(E\ulcorner M\urcorner) \longrightarrow \begin{cases}\text { True } & \text { for } M \in \mathrm{S} \\ \text { False } & \text { otherwise }\end{cases}
$$

Let $M_{0}$ be any non-standard fixed-point combinator, with encoding $\left\ulcorner M_{0}\right\urcorner$. Let Turing be a combinator that takes an encoding of a Turing machine $T$, given by $\ulcorner T\urcorner$, and returns the identity combinator $\mathbf{I}$ if $T$ terminates (and diverges if $T$ diverges). Using Turing, we define the combinator $F$ as follows:

$$
F \equiv \lambda t .\left(\text { Turing } t M_{0}\right)
$$

For any Turing machine $T$, the application $(E\ulcorner(F\ulcorner T\urcorner)\urcorner)$ returns True if $T$ terminates, and returns False if $T$ diverges. This decides the Halting Problem for Turing machines, which is of course, not possible. Hence $E$ does not exist, and $\mathrm{S}$ is not recursive.

The set $\bar{S}$ is the set of terms outside the set of non-standard fixed-point combinators, i.e., the set of terms that do not have the infinite extension $\lambda f . f(f(f \cdots))$. We show that $\overline{\mathrm{S}}$ is recursively enumerable by presenting an effective enumeration of its elements. The proof proceeds much like the proof of Theorem 2.1: Once again, we enumerate the set of combinators, carrying each combinator along in some structure (an ordered triple in this case), and filter out those terms that match our criteria. The details are given below.

We make use of the effective surjection $\mathfrak{C}: \mathbb{N} \rightarrow \Lambda^{0}$ from the proof of Theorem 2.1. Let $V_{0}=\emptyset$. For any $j>0$ we define:

$$
\begin{aligned}
C_{j} & \equiv \mathfrak{C}(j) \\
Q_{j} & \equiv\left\langle C_{j},\left\{C_{j}\right\}, j\right\rangle
\end{aligned}
$$

We use this structure to maintain all the terms that can be reached from $C_{j}$ via any finite number of $\beta \eta$-steps, so as we move from $V_{j}$ to $V_{j+1}$, the second projection of each of the triples "grows" to include all the terms that can be reached by an additional $\beta \eta$-step. We now define the set of $F_{j}$ of all triples for which we can now show that the first projection fails to have the infinite extension $\lambda f . f(f(f \cdots))$. We will make use of the effective predicate $N(x)$ as a "filter":

$$
\begin{aligned}
F_{j}= & \left\{\left\langle x_{1}, x_{2}, x_{3}\right\rangle: N(x), \text { for some } x \in x_{2}\right\} \\
N(x)= & \begin{cases}\text { False } & \text { for } x=\lambda f \cdot f^{n}((\lambda x . A) B), \text { modulo } \\
\alpha \text {-equivalence, and for some } & \\
\text { True } B \in \Lambda, n \in\{0\} \cup \mathbb{N} & \text { otherwise }\end{cases}
\end{aligned}
$$

We now remove the structures that correspond to terms that were found not to have the infinite extension $\lambda f . f(f(f \cdots))$ :

$$
W_{j}=V_{j} \backslash F_{j}
$$


We now extend the second projection to include all the terms that can be reached after one additional $\beta \eta$-step:

$$
\begin{aligned}
& W_{j}^{\prime}=\left\{\left\langle x_{1}, x_{2}^{\prime}, x_{3}\right\rangle:\right. \\
&\left.x_{2}^{\prime}=x_{2} \cup\left\{y: x \rightarrow_{\beta \eta} y, \text { for some } x \in x_{2}\right\}\right\}
\end{aligned}
$$

The set $V_{j+1}$ is defined to contain both the new structure $Q_{j}$, as well as the extended triples:

$$
V_{j+1}=\left\{Q_{j}\right\} \cup W_{j}^{\prime}
$$

The enumeration of the set $\overline{\mathrm{S}}$ is obtained by enumerating the first projections in all the triples in $\left\{F_{j}\right\}_{j \in \mathbb{N}}$ according to the ordering induced by $j$, as the primary index, and then by the third projections in each $F_{j}$, as the secondary index.

\section{Acknowledgments}

I am grateful to BRICS for hosting me and for providing a stimulating environment. Thanks are also due to Olivier Danvy, Neil Jones, Menachem Kojman, and Torben Mogensen for their comments and encouragement.

\section{References}

[1] Hendrik P. Barendregt. The Lambda Calculus, Its Syntax and Semantics. North-Holland, 1984.

[2] Alonzo Church. The Calculi of Lambda-Conversion. Princeton University Press, 1941.

[3] Haskell B. Curry, Robert Feys, and William Craig. Combinatory Logic, volume I. North-Holland Publishing Company, 1958.

[4] Hartley Jr. Rogers. Theory of Recursive Functions and Effective Computability. The MIT Press, 1987.

[5] Richard Statman. Some Examples of Non-Existent Combinators. Theoretical Computer Science, 121:441-448, 1993.

[6] Joseph E. Stoy. Denotational Semantics: the Scott-Strachey Approach to Programming Language Theory. The MIT Press Series in Computer Science. MIT Press, 1977.

[7] Alan Turing. The p-functions in $\lambda$ - $k$-conversion. Journal of Symbolic Logic, pages 164-164, 1937. 


\section{Recent BRICS Report Series Publications}

RS-05-1 Mayer Goldberg. On the Recursive Enumerability of FixedPoint Combinators. January 2005. 7 pp. Superseedes BRICS report RS-04-25.

RS-04-41 Olivier Danvy. Sur un Exemple de Patrick Greussay. December 2004. 14 pp.

RS-04-40 Mads Sig Ager, Olivier Danvy, and Henning Korsholm Rohde. Fast Partial Evaluation of Pattern Matching in Strings. December 2005. 22 pp. To appear in TOPLAS. Supersedes BRICS report RS-03-20.

RS-04-39 Olivier Danvy and Lasse R. Nielsen. CPS Transformation of Beta-Redexes. December 2004. ii +11 pp. Superseedes an article to appear in Information Processing Letters and BRICS report RS-00-35.

RS-04-38 Olin Shivers and Mitchell Wand. Bottom-Up $\beta$-Substitution: Uplinks and $\lambda$-DAGs. December 2004.

RS-04-37 Jørgen Iversen and Peter D. Mosses. Constructive Action Semantics for Core ML. December 2004. 68 pp. To appear in a special Language Definitions and Tool Generation issue of the journal IEE Proceedings Software.

RS-04-36 Mark van den Brand, Jørgen Iversen, and Peter D. Mosses. An Action Environment. December 2004. 27 pp. Appears in Hedin and Van Wyk, editors, Fourth ACM SIGPLAN Workshop on Language Descriptions, Tools and Applications, LDTA '04, 2004, pages 149-168.

RS-04-35 Jørgen Iversen. Type Checking Semantic Functions in ASDF. December 2004.

RS-04-34 Anders Møller and Michael I. Schwartzbach. The Design Space of Type Checkers for XML Transformation Languages. December 2004. 21 pp. Appears in Eiter and Libkin, editors, Database Theory: 10th International Conference, ICDT '05 Proceedings, LNCS 3363, 2005, pages 17-36. 